Voix et Images

voixetimages

\title{
Économie et écriture
}

\section{André Brochu}

Volume 1, numéro 1, septembre 1975

\section{Hubert Aquin}

URI : https://id.erudit.org/iderudit/013991ar

DOI : https://doi.org/10.7202/013991ar

Aller au sommaire du numéro

\section{Éditeur(s)}

Les Presses de l'Université du Québec

\section{ISSN}

0318-9201 (imprimé)

1705-933X (numérique)

Découvrir la revue

\section{Citer cet article}

Brochu, A. (1975). Économie et écriture. Voix et Images, 1(1), 125-127.

https://doi.org/10.7202/013991ar d'utilisation que vous pouvez consulter en ligne.

https://apropos.erudit.org/fr/usagers/politique-dutilisation/ 


\section{Économie et écriture}

Le numéro 11 de la revue Critère, Croissance et démesure, présente un intérêt considérable pour tout lecteur soucieux de connaître et de comprendre les problèmes de notre époque. Les articles qu'il rassemble exposent et mettent à la portée des profanes, en ses grandes lignes, la conjoncture socio-économique actuelle. La connaissance des perspectives économiques d'ensemble, celles qui intéressent la planète tout entière, est indispensable aujourd'hui pour la compréhension du développement des économies particulières de l'Occident ou, plus près de nous, de l'Amérique; et par suite, pour l'intelligence de notre situation sociale, politique, voire même culturelle. Dans ce compte rendu, qui n'est pas exhaustif, je m'arrêterai à quelques idées maîtresses susceptibles d'éclairer, tout au moins indirectement, le débat sur les orientations intellectuelles et littéraires présentes.

Au centre de la réflexion économique de notre temps, il y a le thème du «caractère exponentiel de la croissance économique et démographique sur notre planète", exposé par Yves Mongeau dans son article sur «le Club de Rome et ses critiques" (p. 85). La.progression de la population et de la production n'est pas d'ordre arithmétique mais d'ordre géométrique - la prise de conscience de ce phénomène est encore toute récente - et, "contrairement à la croissance linéaire, celle qui suit une courbe d'évolution exponentielle comporte des effets cumulatifs et implique la possibilité de soudaines et prodigieuses embardées en avant" (p. 86), rendant ainsi très problématique l'espoir d'instaurer un contrôle et de parer à la catastrophe. Le célèbre rapport Meadows, commandé par le Club de Rome, a étudié l'interaction de cinq paramètres particulièrement significatifs, tous soumis à une évolution selon une progression géométrique: la population, l'industrialisation (investissements, production industrielle), la pollution, la production agricole (ressources alimentaires) et les ressources naturelles.

En supposant le statu quo des tendances actuelles, l'analyse par ordinateur de l'interaction des cinq facteurs aboutit à la conclusion que "l'expansion démographique et l'expansion économique s'arrêteront au plus tard au cours du siècle prochain, par suite d'une pénurie de matières 
premières " ( $p .88$ ). Si l'on modifie l'hypothèse de départ, en supposant un développement de la production industrielle dû à l'exploitation de l'énergie solaire, on aboutit à la conclusion d'une pollution catastrophique et à un effondrement plus radical encore de la courbe de la population. Mais supposons qu'on maîtrise aussi la pollution, c'est la pénurie alimentaire qui va entraîner la chute de la production industrielle et l'élévation du taux de mortalité. Que le contrôle de la pollution s'établisse, en plus du recyclage des ressources à soixante-quinze pour cent, de la réduction de la pollution à vingt-cinq pour cent du taux de 1970 et du rendement doublé des terres cultivables, c'est l'épuisement des ressources naturelles, l'accumulation de la pollution et, finalement, la décrudescence de la production alimentaire qui s'ensuivront. «En somme, quelles que soient les hypothèses que l'on formule, le résultat demeure sensiblement le même dès que l'on se contente de solutions purement techniques aux problèmes qui se posent. L'écosystème mondial se comporte toujours de la même manière: une croissance exponentielle de la population et des investissements, suivie d'un effondrement" ( $p .90)$.

Le rapport Meadows est contesté dans certains aspects fondamentaux, d'ordre méthodologique et idéologique, mais il n'en reste pas moins le point de départ d'une réflexion d'ensemble sur l'avenir prochain de la planète et des économies nationales (en particulier celles des sociétés fortement industrialisées).

Un autre article, fort intéressant et d'un caractère plus philosophique, de Maurice Lagueux montre que l'idée de démesure, qui s'attachait, pour Aristote et encore pour Boisguillebert (économiste financier du xvilıe siècle), à la notion de commerce - la monnaie favorisant une accumulation de richesses superflues - s'est ensuite déplacée vers la notion de finance, laquelle faisait «miroiter la prétention apparemment folle de multiplier indéfiniment les moyens d'échange sans égard pour les réserves d'or " (p. 18); et enfin, tout récemment, vers la notion de production et non plus d'échange. Les économistes préclassiques se méfiaient de l'or, les économistes classiques en ont fait un étalon (une mesure) et se sont méfiés plutôt du papier monnaie; au xxe siècle, grâce à Keynes, l'or a cessé d'être la norme au profit des «besoins réels de l'économie"; mais la méfiance porte maintenant sur le caractère exponentiel du développement de la production. Lagueux entrevoit un rétablissement de la confiance par la substitution, à l'objectif de production maximale qui a cours présentement, de l'objectif de production optimale.

Ce qui frappe, dans la plupart des articles du numéro, c'est la nécessité qu'on y fait valoir de corriger une activité économique axée sur le pur accroissement des biens et des revenus par l'introduction de facteurs qualitatifs; la nécessité, donc, de refaire un consensus social autour de valeurs philosophiques, morales. Tout se passe, dans l'esprit des auteurs, comme si un renouveau idéologique pouvait arriver à freiner les tendances de l'économie à la démesure. 
La nécessité de corriger le quantitatif par le qualitatif se fait sentir aussi - est-ce un hasard? - au plan même de la méthode: dans l'analyse économique, on sent le besoin de faire une place aux facteurs sociaux et même culturels. On reproche par exemple au rapport Meadows de ne pas tenir compte du "facteur humain"; et à la notion de produit national brut, ou du moins à son utilisation par les politiciens, de confondre la notion quantitative de volume des biens et services avec celle, qualitative, de bien-être social. Par exemple, si chaque Québécois se brisait une jambe, il y aurait une augmentation importante du produit national brut et nos politiciens s'en glorifieraient... Jean Stafford démontre clairement l'utilité de définir des indicateurs sociaux pertinents, appropriés à chaque société particulière.

La réflexion des économistes et des sociologues n'est pas sans trouver de correspondance dans d'autres secteurs du savoir. Ainsi, l'idée d'un emballement de la production, situation particulière au $x x^{e}$ siècle, m'a fait songer, dans le domaine littéraire, à l'importance qu'accordent Julia Kristeva et le groupe de $T e l$ Quel à la notion de productivité signifiante. Je me suis demandé si la notion de pratique textuelle, entendue comme productivité libérée des contraintes du sens (ou de quelque signifié transcendental) ne reflétait pas la situation économique actuelle et si la logique signifiante d'un texte de Sollers, par exemple, ne pouvait pas être décrite, par rapport à la littérature qui le précède, en termes de progression exponentielle: multiplication, presque à l'infini, des rapports établis entre les signifiants, et donc des lectures possibles du texte. D'autre part, l'entreprise de Sollers s'inscrit consciemment dans le contexte d'une crise de la littérature, 'à laquelle elle compte bien contribuer, et cela n'est pas sans rappeler la catastrophe entrevue au terme de la croissance économique et sociale actuelle.

D'autre part, il semble que, dans la théorie comme dans la pratique économiques, se fasse sentir le besoin impérieux d'une intégration de perspectives non proprement économiques, ce qui va dans le sens d'une intégration plus poussée des diverses problématiques de la connaissance. Le même besoin se fait sentir dans la plupart, sinon dans toutes les disciplines aujourd'hui, aussi bien en sciences humaines que dans les sciences exactes. Il est intéressant de remarquer que, au moment où on entend corriger le quantitatif par le qualitatif dans la pensée économique, on trouve un mouvement symétrique et complémentaire dans le secteur culturel avec le développement de procédures de description «scientifiques" (structuralisme, sémiotique).

La nécessité d'un consensus, qui n'est pas synonyme de confusion mais de mise en rapport dialectique, n'existe pas qu'au niveau social ou politique mais aussi au niveau intellectuel: il est temps de mettre fin à la "guerre des herméneutiques " qui est aussi, plus généralement, une guerre des savoirs 'particuliers, et de domestiquer enfin, à tous les niveaux de l'activité humaine, les pulsions de mort au profit du mieux-être collectif. 